\title{
Ataxia Telangiectasia and Rad3-Related Activation by DNA Damage Mitigates Maladaptive Repair after Acute Kidney Injury
}

\author{
Joseph V. Bonventre ${ }^{a, b}, c, d, e$ \\ aDivision of Renal Medicine, Brigham and Women's Hospital, Boston, MA, USA; b Division of Engineering in Medicine, \\ Brigham and Women's Hospital, Boston, MA, USA; 'Department of Medicine, Harvard Medical School, Boston, MA, \\ USA; ${ }^{\mathrm{d}}$ Harvard Stem Cell Institute, Cambridge, MA, USA; 'Laboratory of Systems Pharmacology, Harvard Medical \\ School, Boston, MA, USA
}

\section{Keywords}

YH2AX · Reactive oxygen species - TASCC · Cyclin G1 .

Fibrosis

\begin{abstract}
DNA damage is an important consequence of injury to the proximal tubule. The proximal tubule cell responds to this damage by mounting a DNA damage response (DDR). Two protein kinases, ataxia-telangiectasia mutated (ATM) or ataxia telangiectasia and Rad3-related (ATR), play an important role in this DDR. If efficient, the DDR can lead to repair of the DNA, cell renewal, and return to a healthy state. In many cases, however, especially in the setting of baseline kidney injury, there is incomplete repair. In human chronic kidney disease (CKD) and in human kidney organoids exposed to acute injury, there is increased evidence of DNA damage and activation of ATR. This review focuses on 3 aspects of the DNA damage and response to it: (1) DNA damage and the DDR precipitated by acute injury; (2) protection afforded by the DDR kinase, ATR, in multiple mouse models of acute kidney injury; and (3) downstream effects of genetic inhibition of ATR in the proximal tubule, leading to maladaptive repair, fibrosis, and CKD.

๑) 2021 S. Karger AG, Basel
\end{abstract}

The pathophysiology of kidney injury often involves injury to the tubule epithelial cell, tubular obstruction, endothelial activation, vascular injury, and a vigorous inflammatory response involving leukocyte recruitment [1$3]$. The repair process can be adaptive, which returns the kidney to a state close to the condition it was in before the injury. In many cases, however, the repair is maladaptive [4], where the kidney returns to a state characterized by incomplete repair, persistent response to the initial insult, and enhanced susceptibility to the continued injury. Maladaptive repair often leads to chronic kidney disease (CKD) and in some cases end-stage kidney disease. The pathobiology of the acute injury depends on the location, the length of time that the injurious factors are operant, and the cell types and intracellular mechanisms involved. An important component of the injury is DNA damage, especially when there are predisposing conditions of in-

Contribution from the AKI and CRRT 2021 Symposium at the 26th International Conference on Advances in Critical Care Nephrology, A Virtual/Hybrid Event from San Diego, CA, USA, February 28 to March 5,2021 . This symposium was supported in part by the NIDDK funded University of Alabama at Birmingham-University of California San Diego O'Brien Center for Acute Kidney Injury Research (P30DK079337). karger@karger.com www.karger.com/nef

(C) 2021 S. Karger AG, Base

Karger"
Correspondence to:

Joseph V. Bonventre, jbonventre@bwh.harvard.edu 
creasing age and prior kidney injury, conditions which reduce the cellular ability to reverse additional adverse perturbations. The DNA damage leads to a DNA damage response (DDR) ultimately leading to cell cycle arrest and senescence. In turn, this results in a senescence-associated secretory response which contributes to the pro-inflammatory CKD and ultimately fibrosis. This short review will focus on 3 aspects of the DNA damage and response to it: (1) DNA damage and the DDR precipitated by acute injury; (2) protection afforded by the DDR kinase, ataxia telangiectasia and Rad3-related (ATR); and (3) downstream effects of inhibition of ATR, leading to maladaptive repair.

DNA damage occurs in response to acute injury to the kidney [5, 6] and is quite pervasive in human CKD [7]. In general, with kidney stress induced by ischemia, nephrotoxicants, aging, etc., there is DNA damage which can be produced in response to reactive oxygen species which can derive from a number of sources including generated from within the cell such as related to mitochondrial injury but also produced by inflammatory cells that are recruited to the damaged site. $\gamma \mathrm{H} 2 \mathrm{AX}$ is often used as a marker for DNA damage. In a recently published study [8], we found an overlap between Kidney Injury Molecule-1 (KIM-1) staining and $\gamma \mathrm{H} 2 \mathrm{AX}$ in human CKD, indicating that KIM-1+ injured cells are often characterized by DNA damage. By comparison, in kidney tissue obtained from patients with minimal change disease with little tubule or interstitial disease, there was little $\gamma \mathrm{H} 2 \mathrm{AX}$ or KIM-1 staining.

A normal cellular response to DNA damage is to mount a DDR [9]. This DDR is controlled in large part by 2 proteins, ataxia-telangiectasia mutated (ATM) or ATR. The DDR can lead to repair of the DNA, cell maintenance, cell renewal, and return to a healthy state. If the cell damage is very severe, ATM and ATR can amplify the damage signal and activate cell death pathways. With less severe injury, however, especially in the setting of baseline kidney injury, there is incomplete repair, blocking of the cell cycle, and potentially senescence. The different phases of the cell cycle can be identified histologically [5]. The G2/M phase can be marked by phospho-histone staining, and the $\mathrm{S}$ phase by BrdU uptake. Cells in any stage after entering the cell cycle will express Ki67. The fraction of dividing cells in the Go/G1 phase can be derived by subtracting the cells in the $S$ and $G 2 / M$ phases from the total that are Ki67+.

In response to DNA damage, activation of the ATR and ATM kinases initiate a complex mechanism of signaling pathways that lead to activation of checkpoint

ATR Activation by DNA Damage

Mitigates Maladaptive Repair kinases to stop the cell cycle in an attempt to allow the DNA to repair. Those checkpoints can stop the cell at the G1/S or G2/M phase of the cell cycle. The advantage of having a checkpoint at G1/S is that this prevents the cells from going into the $S$ phase where the DNA damage may be propagated, and prevents cells from progressing to G2/M. Inhibitors of this G1/S transition have been shown to be protective [10]. On the other hand, increased residence at the G2/M transition is associated with a senescence-associated secretory phenotype that is maladaptive. ATM promotes G2/M arrest. We have reported that aristolochic acid added to HK2 cells will increase the fraction of cells in the G2/M phase of the cell cycle. Inhibition of ATM reduced the fraction of aristolochic acid-treated cells in the G2/M stage of the cell cycle [5].

We studied whether ATR was beneficial or detrimental to the repair process and development of fibrosis. ATR protects the genome against $\mathrm{R}$ loops, which are RNA hybridizing with DNA. With CKD there is significant activation of ATR, particularly the phosphorylated activated form of ATR [8]. In control cases of minimal change disease with little tubulointerstitial involvement, there was very little activation of ATR. We have compared the injury response of wild-type mice and mice in which we selectively deleted ATR from the proximal tubules using a Cre-Lox genetic approach in the setting of 3 models of kidney injury [8]. One model was a cisplatin injury model, where we found that there was much more kidney injury in mice in which renal proximal tubule cell ATR was deleted $\left(\mathrm{ATR}^{\mathrm{RPTC}-{ }^{-}-}\right)$. There was much more DNA damage as reflected by $\gamma \mathrm{H} 2 \mathrm{AX}$ staining.

We also evaluated DNA damage and ATR activation in human kidney organoids differentiated from human pluripotent stem cells and exposed to cisplatin. There was a marked increase in $\gamma \mathrm{H} 2 \mathrm{AX}$ and pATR in human organoid proximal tubules as compared to vehicletreated control organoids. When organoids were exposed to cisplatin in the presence of an inhibitor of ATR there were more phospho-histone-H3+ Ki67+ proximal tubule cells in the G2/M phase of the cell cycle. In mice exposed to cisplatin, the ATR $\mathrm{RPTC}-$-- $^{\mathrm{R} \text { animals had }}$ a higher creatinine and BUN at $72 \mathrm{~h}$ than wild-type mice, indicative of more acute dysfunction in response to cisplatin-induced injury. The $\mathrm{ATR}^{\mathrm{RPTC}-/-}$ mice also had much more KIM-1 staining in the cortex and outer medulla.

In another model of injury, ischemia/reperfusion injury (IRI), we also found enhanced DNA damage and 
functional impairment in $\mathrm{ATR}^{\mathrm{RPTC}-/-}$ mice when compared with wild-type controls. The $\mathrm{ATR}^{\mathrm{RPTC}-1-}$ mice had a delayed recovery in serum creatinine and also developed increased aSMA expression and fibrosis at 28 days post-IR. These results reflected the effect of this persistent injury in the setting of loss of protection by not having ATR in the proximal tubule. In a third model, unilateral ureteral obstruction, there was a marked increase in aSMA and fibrosis as early as 7 days after UUO in $\mathrm{ATR}^{\mathrm{RPTC}-/-}$ mice when compared to wild-type mice. There were greater increases in mRNA transcripts of fibrosis-associated proteins, TGF $\beta$, CTGF, $\alpha$ SMA, and fibronectin, in $\mathrm{ATR}^{\mathrm{RPTC}-1-}$ mice than wild-type mice.

Thus, ATR is beneficial to the repair of the DNA damage that occurs with injury in each of these models. Its presence results in recovery which is less fibrotic. On the other hand, when we genetically eliminated proximal tubule ATR [8], this protective effect of ATR was lost, and there was much more DNA damage that persisted. There was then activation of downstream mediators that block the cell cycle, in particular at the G2/M phase resulting in more senescence.

Downstream of the DDR, cyclin G1, p53, p21, and p16 is upregulated, and these result in cell cycle arrest, particularly at the G2/M phase (Fig. 1). G2/M arrest results in upregulation of cell senescence and the senescence-associated secretory program, which generates pro-inflammatory and profibrotic factors. As described previously, cell senescence refers to the state of a stable cell cycle arrest in which proliferating cells become resistant to growth-promoting stimuli. In the $\mathrm{ATR}^{\mathrm{RPTC}-/-}$ kidneys, there is much more senescence, as reflected by $\beta$-gal staining, after IRI when compared to wild-type mice. Cyclin G1 activation further promotes G2/M arrest. Cyclin G1 increases after aristolochic acid or severe bilateral IRI, or unilateral IRI. When cyclin G1 is constitutively knock out genetically this resulted in marked reduction in KIM-1+ and G2/M phase cells and reduction in fibrosis at 6 weeks after aristolochic acid. Cyclin G1 targeting potentially provides a way to reduce the fibrotic response after injury to the kidney. Ultimately there is the development of more TASCCs (Target of Rapamycin [TOR]-Autophagy Spatial Coupled Compartments) [11] that facilitate the production of profibrogenic mediators. These TASCCs are autophagy compartments that stain for the lysosome-associated membrane protein (LAMP) and mTOR+. We have shown that in a number of different conditions-aristolochic acid nephropathy, severe IRI, and unilateral IRI, the

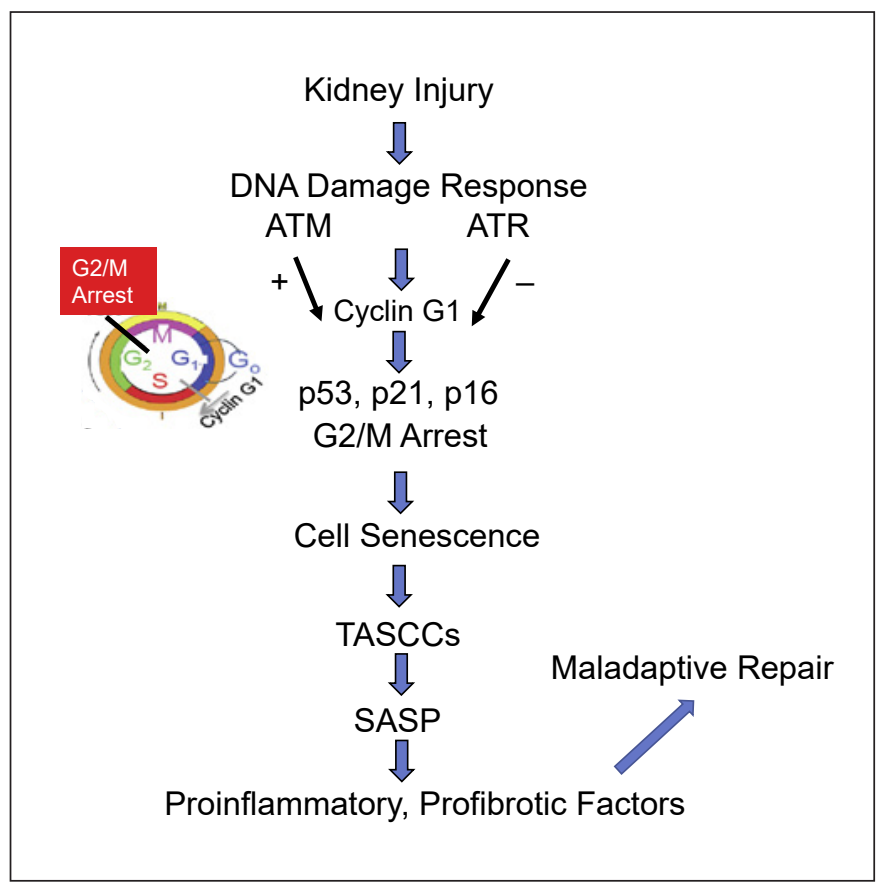

Fig. 1. Kidney DDR after injury. Injury results in DNA damage and activation of a DDR. Two major kinases activated are ATM and ATR. In addition, cyclin G1 is activated. A number of downstream proteins are activated including p53, p21 and p16, and there is cell cycle arrest including arrest at the G2/M phase of the cycle. This arrest then leads to senescence, the generation of TASCCs and the SASP which includes pro-inflammatory and profibrotic factors that act to attract leukocytes and activate myofibroblasts leading to maladaptive repair. DDR, DNA damage response; ATM, ataxiatelangiectasia mutated; ATR, ataxia telangiectasia and Rad3-related; SASP, senescence-associated secretory phenotype.

number of TASCCs are greater than under conditions of less injury, including moderate IRI and conditions of IRI where we introduce protective approaches [11]. Thus, there is an association between the injury and the fibrotic consequences and the appearance of these compartments. TASCCs were also found in 64-day organoids treated with cisplatin for 24 or $120 \mathrm{~h}$.

To summarize, kidney injury leads to DNA damage, and a DDR, with activation of ATM and ATR. There is upregulation of cyclin G1 and downstream molecules which facilitate G2/M arrest, senescence, TASCC formation, and the senescence-associated secretory program which generates pro-inflammatory and profibrotic molecules. These mediators act on pericytes/fibroblasts to differentiate them to myofibroblasts which then secrete additional profibrotic factors ultimately resulting in a fibrotic kidney. 


\section{Conflict of Interest Statement}

J.V.B. is cofounder and holds equity in Goldfinch Bio. He is coinventor on KIM-1 and kidney organoid patents which are assigned to Mass General Brigham. He owns equity in DxNow, Innoviva, Medibeacon, Rubius, Goldilocks, and Verinano and is also a consultant for Angion, Janssen, Praxis, Sarepta, and Seattle Genetics. J.V.B.'s interests were reviewed and are managed by BWH and Mass General Brigham in accordance with their conflict of interest policies.

\section{References}

1 Bonventre JV, Yang L. Cellular pathophysiology of ischemic acute kidney injury. J Clin Invest. 2011 Nov; 121(11):4210-21.

2 Ferenbach DA, Bonventre JV. Mechanisms of maladaptive repair after AKI leading to accelerated kidney ageing and CKD. Nat Rev Nephrol. 2015 May;11(5):264-76.

3 Ferenbach DA, Bonventre JV. Acute kidney injury and chronic kidney disease: from the laboratory to the clinic. Nephrol Ther. 2016 Apr;12(Suppl 1):S41-8.

4 Bonventre JV. Maladaptive proximal tubule repair: cell cycle arrest. Nephron Clin Pract. 2014;127(1-4):61-4

\section{Funding Sources}

J.V.B. was supported by the National Institutes of Health Grants R37DK039773 and R01DK072381.
5 Yang L, Besschetnova TY, Brooks CR, Shah JV, Bonventre JV. Epithelial cell cycle arrest in G2/M mediates kidney fibrosis after injury. Nat Med. 2010 May;16(5):535-43; 1p following 143.

6 Yan M, Tang C, Ma Z, Huang S, Dong Z. DNA damage response in nephrotoxic and ischemic kidney injury. Toxicol Appl Pharmacol. 2016 Dec 15;313:104-8.

7 Schupp N, Stopper H, Heidland A. DNA damage in chronic kidney disease: evaluation of clinical biomarkers. Oxid Med Cell Longev. 2016;2016:3592042.

8 Kishi S, Brooks CR, Taguchi K, Ichimura T, Mori Y, Akinfolarin A, et al. Proximal tubule ATR regulates DNA repair to prevent maladaptive renal injury responses. J Clin Invest. 2019 Nov 1;129(11):4797-816.
9 Ciccia A, Elledge SJ. The DNA damage response: making it safe to play with knives. Mol Cell. 2010 Oct 22;40(2):179-204.

10 DiRocco DP, Bisi J, Roberts P, Strum J, Wong KK, Sharpless N, et al. CDK4/6 inhibition induces epithelial cell cycle arrest and ameliorates acute kidney injury. Am J Physiol Renal Physiol. 2014 Feb 15;306(4):F379-88.

11 Canaud G, Brooks CR, Kishi S, Taguchi K, Nishimura K, Magassa S, et al. Cyclin G1 and TASCC regulate kidney epithelial cell G2-M arrest and fibrotic maladaptive repair. Sci Transl Med. 2019 Jan 23;11(476):eaav4754. 